\title{
ESPECIFICAÇÃO DE PRODUTOS DE SOFTWARE COM FOCO NA EXPERIÊNCIA DO USUÁRIO
}

\author{
Genilda Oliveira de Araujo (UFSC) genilda@gmail.com \\ Lizandra Garcia Lupi Vergara (UFSC) 1.vergara@ufsc.br
}

\section{Resumo}

Os recentes avanços da computação têm sido usados como base para melhoria de processos e oferta de valor diferenciada. Nesse sentido, conceber um produto de software vai além de propor funções e interfaces. Envolve o tratamento de práticas suportadas por computador. Neste âmbito, este artigo apresenta uma abordagem para especificação de produtos de software com base na relação na relação entre atividade e funções. Tal abordagem contempla as dimensões afetiva, racional e sensório-motora do uso do produto, permitindo o tratamento holístico da UX. Sua construção foi baseada nas teorias da atividade e de affordances. Sua principal contribuição é a definição de uma estratégia progressiva para descrição da solução, que inclui desde a visão estratégica das necessidades a serem atendidas, chegando até a materialização do produto em termos de sua interface.

Palavras-Chaves: metodologia de projeto do produto, experiência do usuário, affordances, teoria da atividade.

\section{Introdução}

Os recentes avanços da computação têm sido usados como base para concepção de sistemas capazes de melhorar a forma de prestação de serviços e de estruturação das atividades de trabalho. Como exemplos, pode-se citar o Nubank no setor financeiro, o QuintoAndar na locação de imóveis, a Loggi na entrega expressa, o Hotmart nos cursos online, bem como a MaxMilhas na compra de passagens aéreas. Em todos os casos, há um enfoque na melhoria de processos para oferecer uma entrega de valor diferenciada.

Nesse sentido, conceber um produto de software vai além de propor funções e interfaces. Conforme Kuutti e Bannon (2014), a interação-homem computador (IHC) tem foco na abordagem das práticas suportadas por computador. Segundo Hassenzahl et al. (2013), a prática que permite realizar uma atividade é a primeira arena para o design, pois pode ser moldada por meio do produto para estar mais conectada à satisfação de necessidades e, 
consequentemente, tornar-se mais propensa a oferecer experiências positivas. Assim, o problema de projeto tratado não é a criação do produto de software em si, mas a proposição de formas de ação capazes de potencializar a satisfação de necessidades.

Com base nesse entendimento, pode-se considerar que especificar um produto de software significa especificar uma prática a ser aplicada pelo usuário. Apesar disso, muitas especificações de software tem um caráter funcional e técnico. Descrevem o que o produto faz, bem como os detalhes para sua implementação (Miedema et al., 2007). Desta forma, conceituam o produto de forma isolada e estática, sem fazer referência ao usuário e à atividade a que dará suporte. A consequência disso é que as necessidades motivadoras do uso e os planos de ação gerados para atender a essas necessidades ficam implícitos, permitindo que se perca de vista o problema de fato abordado. Além disso, tais especificações têm normalmente um caráter pragmático e não abordam como o produto dará suporte à dimensão afetiva da atividade, essencial para o tratamento da experiência do usuário (UX).

Considerando essas questões, o presente artigo apresentou uma abordagem para especificação de produtos de software com base na relação entre atividade e funções. Tal abordagem contempla as dimensões afetiva, racional e sensório-motora do uso do produto, permitindo o tratamento holístico da UX. Sua construção foi baseada nas teorias da atividade e de affordances. Sua principal contribuição é a definição de uma estratégia progressiva para descrição da solução, que inclui desde a visão estratégica das necessidades a serem atendidas, chegando até a materialização do produto em termos de sua interface.

\section{Fundamentação teórica}

Esta seção traz uma visão geral dos desafios para especificação de produtos de software e algumas teorias que podem oferecer suporte neste processo.

\subsection{Especificação de produtos e seus desafios}

Uma especificação descreve uma solução de design concebida. Segundo Marai (2018), não consiste em um mero conjunto de requisitos. Mais que isso, reflete o entendimento da equipe de design sobre como os requisitos devem ser tratados. Em outras palavras, é o resultado de um processo de análise e concepção. Em termos práticos, constitui um importante marco no 
projeto por sua dupla função. A primeira é validar com os stakeholders a solução visualizada. A segunda é servir de guia para o processo posterior de implementação do software.

Neste âmbito, o conceito de função é amplamente usado. Segundo Ciavola et al. (2015), funções consistem em declarações que descrevem o que o produto deve fazer em termos de transformações de materiais e de informação. Uma limitação dessa abordagem, conforme Gabelloni et al. (2011), é a redução do poder explicativo da especificação, pois ao tratar as funções isoladas dos usuários e da sua atividade, não é possível descrever seu papel nos sistemas de informação. Nesse sentido, os autores destacam a importância de alcançar um entendimento relacional, permitindo visualizar as conexões entre usuários e produto no uso.

Outra abordagem de especificação são cenários. Segundo Carroll (2000), um cenário é uma história sobre pessoas e suas atividades. Narra episódios de uso imaginados e serve para orientar o desenvolvimento do produto que dará suporte a estas experiências de uso (Rosson e Carroll, 2012). Desta forma, descreve o que o usuário irá fazer com o produto e como irá interagir com ele (Miedema et al., 2007). Neste caso, a dificuldade é encontrar uma representação adequada. Conforme Sutcliffe (2003), as representações variam desde descrições textuais em linguagem natural até representações estruturadas em linguagem formal. O primeiro extremo é amplamente usado, pois a linguagem natural favorece a abordagem da subjetividade da experiência. No entanto, a ausência de estrutura exige expertise para elaborar um texto que aborde os múltiplos aspectos da experiência.

\subsection{Teoria da atividade}

Esta pesquisa trata a descrição da prática subjacente ao produto com base na teoria da atividade. De modo específico, é usada a estrutura da atividade (Leont'ev, 1978). Esta estrutura, mostrada na figura 1, é um modelo hierárquico composto por três níveis de abstração que abordam as dimensões afetiva, racional e sensório-motora da atividade.

O nível mais alto de abstração foca na atividade como um todo e aborda o seu propósito, que consiste na satisfação de necessidades. Estas necessidades dão origem à atividade e atuam como seu motivo energizante (porque). Segundo Leont'ev (1978), a função do motivo é ajudar o indivíduo a avaliar as circunstâncias e suas ações, dotando-as de significado e afeto. No segundo nível, estão as ações, que são os processos que realizam a atividade. São planejadas a partir da identificação de uma meta geral capaz de satisfazer à necessidade (o que fazer). Por fim, no terceiro nível, cada ação pode ser decomposta em uma ou mais operações, que 
estabelecem os passos concretos para sua execução física (como fazer). Estas operações não são planejadas pelo indivíduo, mas realizadas de acordo com as condições dadas pelo contexto, que inclui os produtos usados. Por exemplo, ao pesquisar uma passagem aérea, a forma de indicação da data de partida será feita de acordo com os componentes da interface. Estas condições são detectadas e tratadas pelo sistema sensório-motor (Leont'ev, 1981).

Figura 1 - Estrutura da atividade
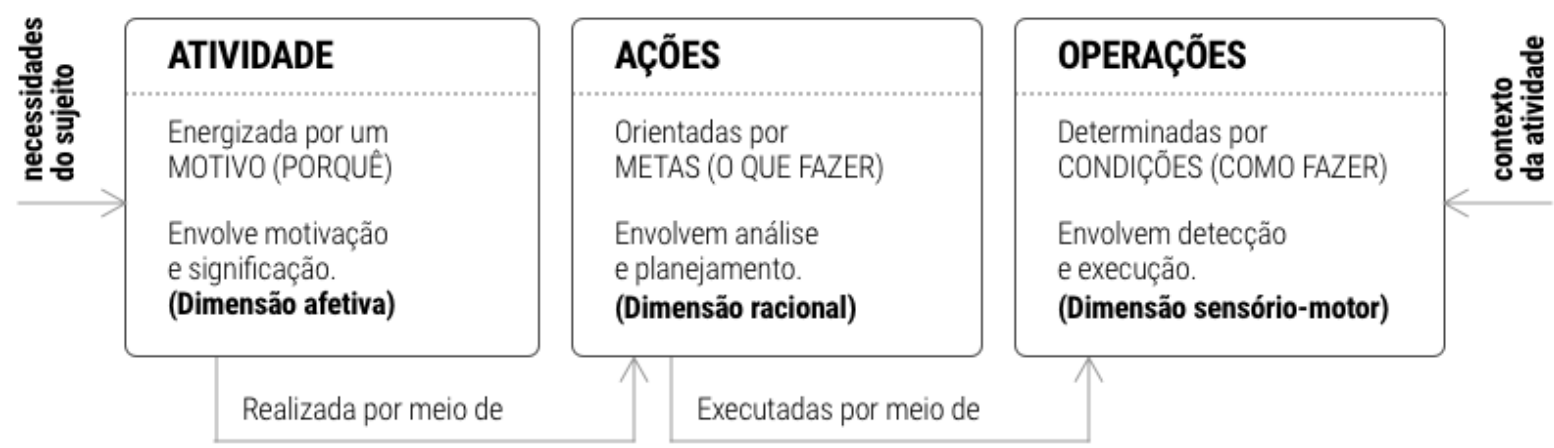

Fonte: elaborada pelos autores

Uma vantagem desta estrutura é seu poder retórico, resultante de uma nomenclatura que aborda construções conceituais presentes em qualquer sistema interativo (Halverson, 2002). Assim, permite o mapeamento do domínio de problema. Para Marsh e Nardi (2014), pode ser aplicada em uma abordagem narrativa dos problemas de design, permitindo planejar, descrever, desenvolver e avaliar cenários interativos. Em especial, sua característica hierárquica permite abordar desde aspectos de alto nível referentes a motivações e necessidades, até aspectos básicos da interação física com a interface (Marsh, 2010). Além disso, para ele, o nível intermediário das ações concentra o coração da narrativa, permitindo descrever o que as pessoas fazem.

Do ponto de vista da abordagem da UX, essa estrutura ajuda a tratar as diferentes dimensões de experiência que o usuário vivencia durante o uso de um produto (Von Saucken et al., 2012). De acordo com a norma 9241-11 (ISO, 2018), a UX inclui "todas as emoções, crenças, preferências, percepções, respostas físicas e psicológicas dos usuários, comportamentos e realizações que ocorrem antes, durante e após o uso". Ou seja, envolve as dimensões afetiva, cognitiva e sensório-motora da atividade. 


\subsection{Affordances}

A conexão do produto com a atividade a com os usuários é tratada com base em affordances. Segundo Mettler e Wulf (2019), affordances são potenciais para ação que decorrem da relação entre um produto e um indivíduo em atividade. Estes potenciais habilitam e restringem o que o usuário pode fazer por meio do produto (Hutchby, 2001). Segundo Gibson (1979), affordances não são propriedades dos produtos. Surgem da relação entre as características dos usuários e as características do produto e do contexto. Nesse sentido, o design baseado em affordances insere o conceito de função em uma imagem mais ampla e sofisticada, incluindo os recursos físicos e as intenções, percepções e ações do usuário (Gabelloni et al., 2011).

Um aspecto interessante a se destacar é que esses relacionamentos ocorrem em diferentes dimensões. Segundo Bærentsen e Trettvik (2002), é possível distinguir uma categoria de affordances para cada nível da estrutura da atividade. Nesse sentido, a lente de affordances ajuda a descrever aquilo que um produto deve oferecer nas dimensões afetiva, racional e sensório-motora. Ou seja, trata dos requisitos que precisam ser atendidos pelo produto para um suporte holístico à UX. Conforme destacam Galvao e Sato (2005), requisitos em termos de affordances contribuem para a tomada de melhores decisões acerca da arquitetura do produto.

\section{Metodologia}

Este trabalho parte do pressuposto que affordances podem ser usados para a descrição de requisitos, servindo de base para a descrição funcional. Também considera que a abordagem da UX precisa ser holística, incluindo as dimensões afetiva, racional e sensório-motora. Assim, para desenvolver a abordagem de especificação proposta, primeiramente foi elaborado um modelo teórico que descreve os potenciais que um produto deve oferecer em cada dimensão da atividade.

A base para o modelo teórico foi uma revisão sistemática acerca de categorias de affordances. Estas categorias foram usadas para identificar os potenciais a serem providos nas diferentes dimensões da atividade. O protocolo para a revisão foi criado com base na metodologia de Kitchenham e Charters (2007). A estratégia de busca definida foi aplicada nas bases SCOPUS, ACM Digital Library e Web of Science. Foram usadas duas buscas para identificar categorias de affordances. A primeira visou identificar esquemas classificatórios baseados na 
teoria da atividade. A string continha as palavras-chave ("activity theory") AND ("affordances" or "affordance"). A segunda visou identificar esquemas classificatórios não são baseados na teoria da atividade. A string de busca usada foi ("affordances" OR "affordance") AND ("model" OR "framework" OR "approach" OR "classification" OR "typology" OR "categories" OR "types" OR "levels" OR "classes" OR "taxonomy") AND ("human computer interaction" or "product design"). Foram incluídos artigos tanto de revistas quanto de conferências, sem limitação do período de publicação.

\section{Resultados}

\subsection{Modelo teórico}

A partir dos trabalhos identificados na literatura, foi sintetizado um modelo com as categorias de affordances que um produto deve oferecer para potencializar a atividade e a UX:

- Oportunidades de experiência: associadas ao nível da atividade, representam o potencial que o uso do produto tem de satisfazer as necessidades que dão origem à atividade (Bærentsen e Trettvik, 2002). Refletem a adequação do produto ao propósito da atividade (Turner e Turner, 2002);

- Oportunidades de realização de tarefas: associadas ao nível das ações, representam o potencial que as funcionalidades do produto têm de permitir a realização de tarefas e o alcance de metas (Jonietz e Timpf, 2013; Sharritt, 2010). Estão ligados à realização de algo por meio do produto (Grange e Benbasat, 2011; Turner e Turner, 2002);

- Oportunidades de manipulação: associadas ao nível operacional, representam o potencial que a interface do produto tem de ser manipulada considerando as condições de uso (Pols, 2012). Permitem o usuário agir fisicamente sobre o produto (Ciavola e Gershenson, 2016; Kaptelinin e Nardi, 2012) para a ativação de suas funções (Grange e Benbasat, 2011).

\subsection{Abordagem de especificação}

A partir do modelo teórico, foi elaborada a abordagem de especificação mostrada na figura 2. Seus passos estão organizados em uma matriz que permite visualizar o que se relaciona ao tratamento de cada uma das dimensões da atividade: afetiva, racional e sensório-motora. Além disso, descreve duas camadas de especificação. A primeira aborda as oportunidades, ou 
seja, affordances, que se deseja disponibilizar para o suporte à atividade. A segunda trata dos elementos da arquitetura do produto necessários para que as oportunidades possam estar disponíveis.

Figura 2 - Abordagem de especificação proposta

PARA CADA GRUPO DE USUÁRIOS DO SISTEMA (PAPEL)

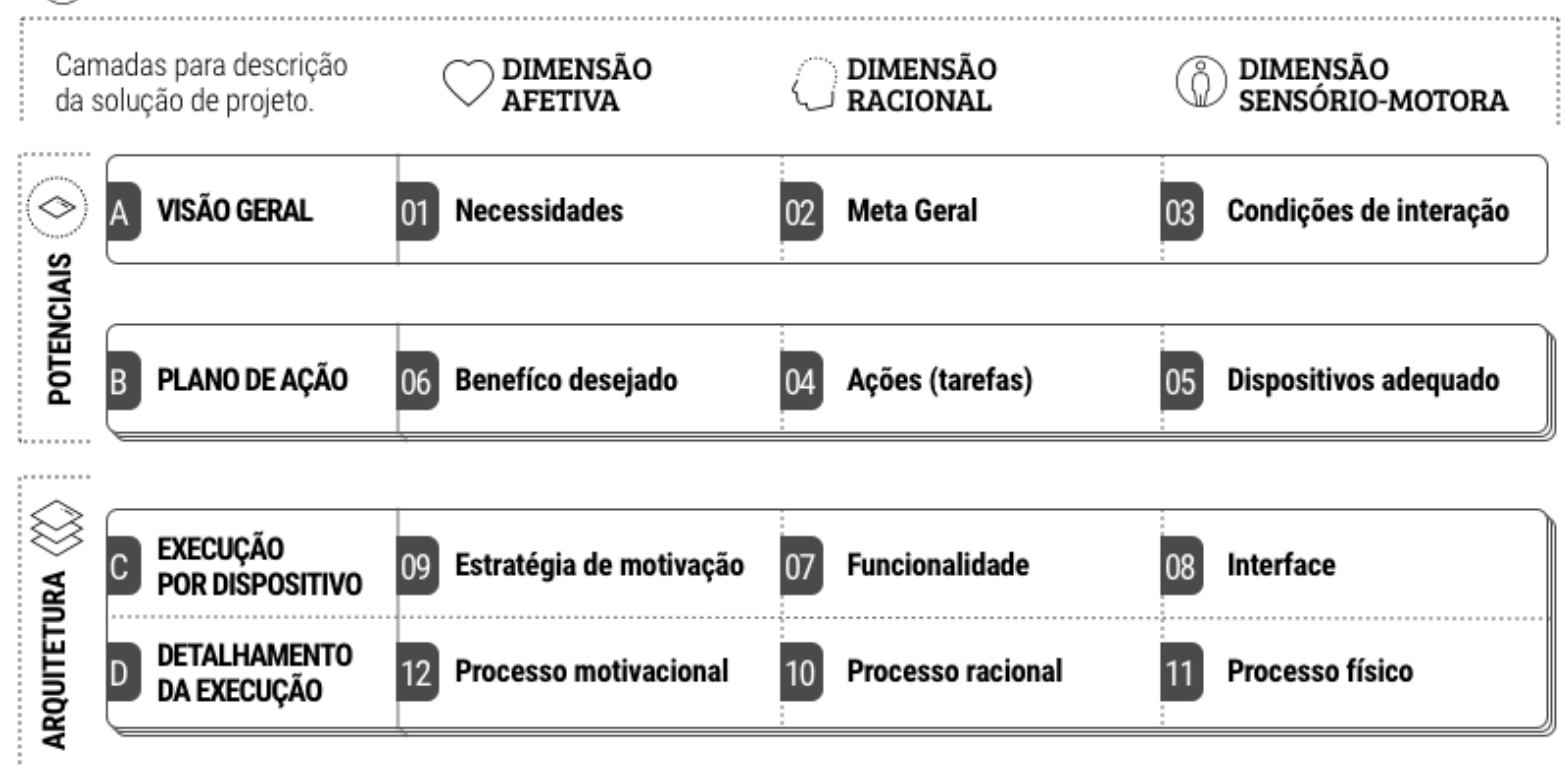

Fonte: elaborada pelos autores

O primeiro passo para a aplicação da abordagem é identificar o grupo de usuários que o aplicativo irá atender. Um sistema pode oferecer suporte a diferentes grupos, cada qual ocupando um papel na atividade. Por exemplo, no aplicativo 99, há usuários como passageiros e outros como motoristas. Cada um deles traz demandas específicas para o produto e por isso, representam especificações diferentes.

Após a identificação do grupo de usuários, pode-se iniciar a especificação das oportunidades. Esse processo consiste em criar uma descrição da atividade em alto nível, chamada de visão geral (A) que incorpora necessidades (1), meta geral (2) e condições de interação (3). Necessidades descrevem aquilo que um indivíduo, uma organização ou um grupo social desejam obter com a atividade. Estão relacionadas a vitalidade, bem-estar e crescimento (Zhang, 2008) e incluem aspectos como autonomia, autoimagem, competência, sucesso, liderança, influência, segurança e conexão com outros (Tang e Zhang, 2018). A meta geral descreve o que será feito pelo usuário para satisfazer as necessidades. Embora essa meta sirva para direcionar a ação de um grupo específico de usuários, pode estar alinhada e dar suporte a metas organizacionais e sociais. Por fim, na dimensão sensório-motora, devem ser descritos 
os recursos disponíveis para que o usuário execute sua meta de ação. Por exemplo, pode-se identificar os tipos de dispositivos, bem como as condições físicas do usuário e do ambiente. Como exemplo dessa etapa, pode-se pensar em um usuário com a necessidade de descanso, que define como meta planejar uma viagem para uma região praiana e pode fazer uso de seu desktop ou smartphone para executar a meta.

O próximo passo é o detalhamento das oportunidades, que envolve a descrição do plano de ação (B) usado para alcançar a meta geral. Segundo Ciavola e Gershenson (2016), um plano de ação é uma hierarquia de metas, que inclui uma meta geral e um conjunto de submetas paralelas ou sequenciais. Cada submeta corresponde a uma ação ou tarefa (4). Conforme os autores, planos podem ter a forma de listas de ações que permitem documentar, examinar, comparar e otimizar os processos de uso. Dando continuidade ao exemplo, a meta geral de planejar a viagem pode ser decomposta nas submetas de comprar passagem aérea e reservar hotel. Por sua vez, cada uma delas pode ser decomposta em outros níveis. Por exemplo, a compra de passagem envolve as ações de encontrar opções de voo e efetuar pagamento. Para detalhar a dimensão sensório-motora, deve-se descrever os dispositivos compatíveis (5) com cada ação. Na dimensão afetiva, deve-se descrever o benefício desejado (6) com a ação. Por exemplo, encontrar opções de voo permite planejar agenda e gastos da viagem. Por sua vez, efetuar o pagamento permite confirmar planejamento. Em ambos os casos, o usuário pode usar tanto desktop quanto smartphone.

A partir da descrição das oportunidades, pode-se descrever a arquitetura do produto. Conforme Gabelloni et al. (2011), cada ação pode ser traduzida em uma ou mais funções (7). Por exemplo, para dar suporte à ação de encontrar opções de voos, pode-se oferecer a função de busca, que apresenta uma lista de resultados com dados completos de voos e tarifas. Como alternativa, pode-se também oferecer a função de sugestão de voos, com base nas promoções existentes. Desta forma, a separação entre oportunidades e funções abre espaço para geração de diferentes alternativas. Focando na estimulação do usuário, deve-se descrever uma estratégia de motivação (8) para cada função. Seu propósito é facilitar que o usuário identifique o benefício que será gerado. Por exemplo, na apresentação dos resultados da busca, pode-se destacar o voo com o menor preço. É importante observar que pode haver variações nas funções entre os dispositivos. Por exemplo, a lista de resultados da busca pode ter um formato detalhado no desktop e resumido no smartphone. Desta forma, pode-se identificar essas variações na lista de funções e definir o dispositivo alvo (9) de cada uma. 
Por fim, passa-se à etapa de detalhamento da execução (D). Nela, cada função pode ser detalhada como um processo racional que descreve o procedimento para realizar a tarefa (10). Consiste em um passo a passo contendo as informações e tomadas de decisão do usuário. Por exemplo, a função de busca requer que o usuário defina data de partida, data de retorno, origem, destino e número de passageiros. Na dimensão sensório-motora, o processo racional ganha estrutura concreta com base em uma interface que define um processo físico (11) de manipulações dos componentes interativos. Por exemplo, a forma física de indicação da data de partida pode envolver digitação ou seleção em um calendário. No nível do processo físico, sugere-se evitar especificação textual e adotar prototipação. Por fim, deve-se trabalhar o processo motivacional (12), buscando relacionar os resultados obtidos com o benefício esperado. A percepção dessa relação gera gratificações nos usuários (Sharritt, 2010), ou seja, experiências positivas, pois está ligada ao prazer de ter suas necessidades atendidas. Este nível pode ser trabalho com mensagens de feedback, imagens ou elementos de gamificação (Tang e Zhang, 2018).

\section{Considerações finais}

O presente artigo apresentou uma abordagem para especificação de produtos de software com base na relação entre atividade e funções. Tal descrição contempla as dimensões afetiva, racional e sensório-motora do uso do produto, permitindo o tratamento holístico da UX. Sua principal contribuição é definir uma estratégia progressiva para descrição da solução. É uma abordagem top-down convergente com o modelo de Garrett (2011) sobre os cinco planos de abstração nos quais as decisões de projeto são tomadas. No primeiro plano, chamado de estratégia, são definidas as necessidades atendidas pelo produto. $\mathrm{Na}$ abordagem proposta, este plano corresponde à etapa de visão geral dos potenciais a serem providos. O segundo plano, de escopo, envolve a seleção das funcionalidades para atender à estratégia. Corresponde à definição do plano de ação e seu mapeamento em termos de funções. No terceiro plano, o da estrutura, ocorre a definição da arquitetura de informação e dos fluxos de interação para realização de tarefas. Tal plano corresponde ao detalhamento do processo racional. No quarto plano, esqueleto, é definida a interface, que corresponde ao detalhamento do processo físico. Por fim, no plano da superfície, é feito o tratamento gráfico dos componentes da interface, relacionado ao processo físico e motivacional. Desta forma, abordagem proposta inclui desde a visão estratégica das necessidades, chegando até a materialização do produto em termos de sua interface. Por fim, como sugestão de trabalhos futuros, pode-se citar a elaboração de 
heurísticas que apoiem a tomada de decisão em cada etapa da especificação, bem como a avaliação da qualidade do produto de software.

\section{REFERÊNCIAS}

BÆRENTSEN, K. B.; TRETTVIK, J. An activity theory approach to affordance. Proceedings of the second Nordic conference on Human-computer interaction. Anais...Arhus, Denmark: ACM, 2002.

CARROLL, J. M. Five reasons for scenario-based design. Interacting with computers, v. 13, n. 1, p. 43-60, 2000.

CIAVOLA, B. T.; GERSHENSON, J. K. Affordance theory for engineering design. Research in Engineering Design, v. 27, n. 3, p. 251-263, 2016.

CIAVOLA, B. T.; WU, C.; GERSHENSON, J. K. Integrating Function- and Affordance-Based Design Representations. Journal of Mechanical Design, v. 137, n. 5, 2015.

GABELLONI, D. et al. On the link between features and functions. DS 68-2: Proceedings of the 18th International Conference on Engineering Design (ICED 11), Impacting Society through Engineering Design, Vol. 2: Design Theory and Research Methodology, Lyngby/Copenhagen, Denmark, 15.-19.08. 2011. Anais...2011.

GARRETT, J. J. The Elements of User Experience: User-centered Design for the Web and Beyond. [s.1.] New Riders, 2011.

GIBSON, J. J. The Ecological Approach to Visual Perception. [s.1.] Houghton Mifflin, 1979.

GRANGE, C.; BENBASAT, I. A guiding framework for developing theories investigating the design drivers of IT use and value. Proceedings of the JAIS Theory Development Workshop. Anais...2011.

HALVERSON, C. A. Activity Theory and Distributed Cognition: Or What Does CSCW Need to DO with Theories? Computer supported cooperative work: CSCW: an international journal, v. 11, n. 1-2, p. 243267,2002

HASSENZAHL, M. et al. Designing Moments of Meaning and Pleasure. Experience Design and Happiness. International Journal of Design, v. 7, n. 3, p. 21-31, 2013.

HUTCHBY, I. Technologies, Texts and Affordances. Sociology, v. 35, n. 2, p. 441-456, 2001.

ISO. Ergonomics of human-system interaction-part 11: Usability: Definitions and concepts. [s.1.] International Organization for Standardization, 2018.

JONIETZ, D.; TIMPF, S. An Affordance-Based Simulation Framework for Assessing Spatial Suitability. Proceedings of the 11th International Conference on Spatial Information Theory - Volume 8116. Anais...: COSIT 2013.New York, NY, USA: Springer-Verlag New York, Inc., 2013.

KAPTELININ, V.; NARDI, B. Affordances in HCI: toward a mediated action perspective. Proceedings of the SIGCHI Conference on Human Factors in Computing Systems. Anais...ACM, 2012.

KITCHENHAM, B.; CHARTERS, S. Guidelines for performing Syst Software Engineeri. [s.1.] Keele University and Durham University Joint Report, 2007.

KUUTTI, K.; BAnNON, L. J. The Turn to Practice in HCI: Towards a Research Agenda. Proceedings of the SIGCHI Conference on Human Factors in Computing Systems. Anais...: CHI '14. New York, NY, USA: ACM, 2014.

LEONT'EV, A. N. Activity, Consciousness, and Personality. Englewood Cliffs: Prentice-Hall, 1978. 
LEONT'EV, A. N. Problems of the Development of Mind. Moscow: Progress, 1981.

MARAI, G. E. Activity-Centered Domain Characterization for Problem-Driven Scientific Visualization. IEEE transactions on visualization and computer graphics, v. 24, n. 1, p. 913-922, 2018.

MARSH, T. Activity-based scenario design, development, and assessment in serious games. Gaming and cognition: Theories and practice from the, p. 213-225, 2010.

MARSH, T.; NARDI, B. Spheres and Lenses: Activity-Based Scenario / Narrative Approach for Design and Evaluation of Entertainment through Engagement (Y. Pisan, N. M. Sgouros, \& T. Marsh, Eds.) Entertainment Computing - ICEC 2014. Anais...: Lecture Notes in Computer Science. In: INTERNATIONAL CONFERENCE ON ENTERTAINMENT COMPUTING. Springer Berlin Heidelberg, 2014.

METTLER, T.; WULF, J. Physiolytics at the workplace: Affordances and constraints of wearables use from an employee's perspective. Information Systems Journal, v. 29, n. 1, p. 245-273, 2019.

MIEDEMA, J. et al. Synergy of Technical Specifications, Functional Specifications and Scenarios in Requirements Specifications. In: The Future of Product Development. [s.1.] Springer, Berlin, Heidelberg, 2007. p. 235-245.

POLS, A. J. K. Characterising affordances: The descriptions-of-affordances-model. Design Studies, v. 33, n. 2, p. 113-125, 2012.

ROSSON, M.; CARROLL, J. Scenario-Based Design: Fundamentals, Evolving Technologies, and Emerging Applications, Third Edition. In: JACKO, J. (Ed.). Human-Computer Interaction Handbook. Human Factors and Ergonomics. [s.1.] CRC Press, 2012. v. 20126252p. 1105-1124.

SHARRITT, M. J. Evaluating Video Game Design and Interactivity. In: VAN ECK, R. (Ed.). . Interdisciplinary Models and Tools for Serious Games. [s.1.] IGI Global, 2010. p. 177-205.

SUTCLIFFE, A. Scenario-based requirements engineering. Proceedings. 11th IEEE International Requirements Engineering Conference, 2003. Anais...2003.

TANG, J.; ZHANG, P. Gamification and Basic Human Needs in Information Technology Design: A Literature Analysis. Proceedings of the 3rd International Conference on Crowd Science and Engineering ICCSE' 18. Anais...: ICCSE'18. In: THE 3RD INTERNATIONAL CONFERENCE. New York, New York, USA: ACM Press, 2018.

TURNER, P.; TURNER, S. An Affordance-based Framework for CVE Evaluation. In: XRISTINE FAULKNER B A PGCE; JANET FINLAY BA, M.; DÉTIENNE, F. (Eds.). People and Computers XVI - Memorable Yet Invisible. [s.1.] Springer London, 2002. p. 89-103.

VON SAUCKEN, C. et al. Customer experience interaction model. Traducao ITS d.o.o.; HSM informatika d.o.o.; OSA Softver d.o.o.; Print Studio d.o.o.; RENAULT NISSAN HRVATSKA d.o.o.; CADLab FSB. [s.l: s.n.]. v. DS 70 .

ZHANG, P. Motivational affordances: reasons for ICT design and use. Communications of the ACM, v. 51, n. 11, p. 145-147, 2008. 